in an early stage of the evolution of modern man (Homo sapiens). Unfortunately, there was nothing found with the bones of Rhodesian man which tells us at what point in the pleistocene period he lived; we infer from the circumstances in which he was found that he is at least mid-pleistocene in date. Some light has been thrown on this and other problems relating to the history of early man in Africa by Dr. Louis Leakey's researches. Dr. Leakey led three expeditions to East Africa between 1925 and 1932, in the course of which he demonstrated the existence of implement-containing deposits covering the greater part of the pleistocene period. Near the base of these deposits was discovered a fragment of a human lower jaw -which has characters not unlike those we expect to find in Rhodesian man. In strata of later but of uncertain date at Kanjera, Dr. Leakey found cranial fragments of the most primitive type of Negro known to us. At first sight, the difference between the Rhodesian and Kanjera types of humanity seems very great, and yet in the opinion of the writer it is possible to conceive the evolution of the Kanjera Negro from Rhodesian man. Whether this evolution has taken place or not is one of the many problems which students of fossil man may hope to solve in the near future.

When King George came to the throne our knowledge of ancient man was almost entirely confined to Europe. Java was the only exception. Discoveries of fossil man of extinct types have now been made in every continent of the world. Every large land area-Europe, Asia, Africa and Australasia-has yielded fossil remains of human types totally different from any now living-all save America. No evidence has been found of the existence in the New World of a type of humanity that differs materially from types which still exist in America.

\title{
Discovery and Significance of Vitamins
}

\section{By 'Sir Frederick Gowland Hopkins, P.R.s., Sir William Dunn Professor of Biochemistry, University of Cambridge}

UNTIL the end of the first decade of the present century, official teaching concerning the nutritional needs of the human body was still based on the results of classical studies by Carl Voit and Max Rubner and on the views of the Munich School thence derived. The adequacy of a dietary was measured in terms of calories and protein alone. It was generally believed, alike by the academic physiologist and by those concerned with practical dietaries, that, questions of palatability and digestibility apart, so long as the food of an individual provided sufficient potential energy for the activities of his internal organs and for the external mechanical work he might be called upon to do, the only demand of a more specific kind made by his body was for a certain, rather ill-defined, minimum of protein, to subserve the growth and maintenance of its tissues. Beside the carbohydrates, fats and proteins which provide these essentials, natural foods were known, of course, to contain a variety of other substances. These, however, are present individually in very small amount, and except for certain minerals among them, necessary for the formation of bone and for the maintenance of particular physical conditions in the body, they assumed to be without nutritional importance.

Facts, nevertheless, were already known which might well have suggested that the body makes calls upon its food to supply needs more subtle and more specific than those thus recognised. The history of scurvy, for example, and the clear demonstration, made already in the eighteenth century, of the dramatic cure of that fell disease which follows upon suitable, though relatively very small, additions to an errant dietary, should, it would seem, have provided a strong suggestion for the existence in certain foods of a substance small in amount but with highly specific properties essential for the support of normal nutrition; that is, for the existence of what we now define as a vitamin. But unfortunately, the views of the majority concerning the influence of anti-scorbutic foods remained for many years vague and obscure. It was attributed to such qualities as 'freshness' without further analysis of these qualities, or to known constituents without proof of their efficacy. True, so far back as 1841, an American physician, G. Budd, had ascribed the action of such foods "to an essential element which it is hardly too sanguine to state will be discovered by organic chemistry or the experiments of physiologists in a not far distant future". Had organic chemists or physiologists been then stimulated by this objective view to seek for a definite substance in such well-known anti-scorbutic materials as, say, lemon or orange juice-a substance which when isolated could display by itself the anti-scorbutic powers of these fruits-it is likely that a realisation of the significance of vitamins might have come 
long ago ; but current thought concerning nutrition was not yet prepared to profit from such suggestions.

Scurvy, of course, is now recognised as one of a group of so-called deficiency diseases-pathological conditions in each of which a group of symptoms is displayed, directly due to the lack of some necessary nutritional factor. It was in 1897 that evidence for the existence of another such disease was clearly revealed. Eijkman, a Dutch hygienist, had been led by extensive observations to the belief that the disease beriberi was associated with the consumption by human communities of polished rice as a basal food. He then found that it is possible to produce an illness in fowls similar to beriberi by feeding the birds on polished rice, and he was further able to prevent or cure it by administering an extract of rice polishings. The discovery that the disease could be thus produced and cured experimentally greatly assisted its study; just as the later observation of Holst and Fröhlich that the guinea pig rapidly displays the symptoms of scurvy when placed upon scorbutic diets, while promptly cured by anti-scorbutics, made easy the experimental study of the latter disease and provided a ready biological test for the presence and relative amounts of the curative agent in various foods.

The explanation first offered by Eijkman for the production of beriberi during the consumption of polished rice was to the effect that the condition is a state of intoxication brought about by the consumption of excessive quantities of starch, and that in the so-called 'silver skin' which is removed by polishing, though not in the bulk of the grain, there is a substance which counteracts the toxic products of the disturbed metabolism. This hypothesis was far-fetched and inhibitory, but the conception of disease as the direct result of a specific deficiency in food was foreign to the thought of the time. Later, however, partly owing to the work of others and partly to extended experiments of his own, Eijkman came to the definite conclusion that there is present in rice polishings an individual substance differing from the then known food constituents, but essential to normal nutrition, though required in very small amount. Even before Eijkman himself had come to this final conclusion, the work of others had made it probable, and by 1910 the significant facts had become fully established. Among those whose work contributed to their establishment must be mentioned : Grijns, a countryman of Eijkman; Vedder and Chamberlain, of the American Medical Service; and the British investigators Fraser and Stanton, whose investigations were carried out in the Malay States. All of these helped to prove that the preventitive of beriberi is a definite chemical substance, and the last-mentioned in particular took pioneer steps which were ultimately to lead later workers to a successful isolation of that substance.

Those who worked on beriberi during these years thought and wrote as pathologists, with their attention primarily directed to the causation and cure of a particular disease. Though doubtless the suggestion for an extension of the kind of knowledge gained was ready to hand, as a matter of fact their writings at first contained no reference to the possibility that substances with the properties we now attribute to vitamins might function widely and prove to be necessary for the support of such fundamental physiological processes as growth itself.

This more general and more physiological conception of the functions of vitamins arose directly from the results of feeding animals on experimental diets. If the assumption were right that proteins, fats and carbohydrates, together with essential minerals, are the sole nutritional necessities, then these materials should support all the functions of the body when each of them is supplied in a pure form, no less adequately than when, in natural foods, they are consumed in association with small amounts of many other substances. The nutritional value of such purified materials supplied in artificial dietaries was at one time the subject of many experiments. The results of these were uncertain and contradictory, owing to the fact that purification was often not complete. It was not then realised that substances present in extremely small amount may profoundly affect the value of a diet. It is this circumstance that our present knowledge of vitamins has made so clear.

In 1906-7 the writer engaged in feeding rats upon highly purified materials of the above kind, and found them wholly unable to support health or normal growth, though certain additions, very minute in amount, greatly increased their nutritional adequacy. It happened that yeast extracts were among the addenda which were successful in this respect, but only, as is clear to-day, because the fat employed in these experiments was filtered butter fat. We know now that butter itself contains certain of the essential vitamins, while yeast supplied the others. These experiments confirmed a personal belief in the importance for nutrition of minor constituents in natural foods, and public expression was given to this belief; but the experimental results were not then published.

In the autumn of 1911 the results of later experiments were communicated to the Biochemical Society, and these were published in the following year in a paper which made a general claim for 
the "importance of accessory factors in normal dietaries". Funk at about the same time impressively summarised the then available knowledge concerning deficiency diseases, and proposed the name 'vitamine' for the substance of which a lack might in each case be presumed to produce the pathological condition. On chemical grounds J. C. Drummond suggested that the final ' $e$ ' in Funk's proposed name should be omitted, and this has become customary. By 1912, then, there was fully adequate evidence for the wide importance of vitamins, and from that time progress in their study has been continuous.

Immediately before the War and until near its end, American investigators were the chief contributors to this progress. T. B. Osborne and L. B. Mendel at Yale and E. V. McCollum at Wisconsin (afterwards at Johns Hopkins University) were separately engaged upon nutritional experiments with artificial dietaries. For a little while after the present writer's publication in 1912, these workers were not fully convinced of the necessity for a vitamin supply. Osborne and Mendel believed for some time that they had succeeded in maintaining rats upon purified diets. Soon afterwards conviction came, and important contributions to the subject were made at both centres. In particular, American studies produced at this time proof that vitamins existed in natural foods in different associations, and led to a distinction between 'fat soluble' and 'water soluble' individuals; a distinction which, though in itself not of fundamental importance, greatly helped later developments in the subject, many of which have been due to workers in America.

During the later stages of the War, when many nutritional problems had to be faced, intensive studies began at the Lister Institute in London. These comprised pioneer work by $\mathrm{A}$. Harden and S. S. Zilva, and the important experiments of Harriette Chick and her colleagues, which have continued to the present day. At this time, University College, London, became also a centre of activity owing to the work and influence of J. C. Drummond, while the classical experiments of $\mathrm{E}$. Mellanby on the production of rickets were already in progress. A few years later, interest in the subject penetrated into every European country, and research became everywhere very active. Recently, publications dealing with vitamins have reached a total of a thousand in a single year.

To-day we have knowledge of some eight or nine vitamins, each proved to have its own specific influence in maintaining the normal course of events in the living body, and each exercising its functions when in exceedingly small concentra- tions. Happily the actual chemical constitution of some of them is now known.

It is, of course, impossible in a brief review to recount all the stages of discovery in the case of each of these substances. The existence of individual vitamins, each with its special influence in the body, has in the majority of cases been revealed by the experimental feeding of animals on the following general lines. Natural products or preparations - crude when experiments beganfrom natural sources, animal or vegetable, when simultaneously added in characteristically small amounts to a vitamin-free dietary, were found to render it capable of supporting normal nutrition. The tendency at first was to assume that each effective addendum contained one active ingredient. The next step in progress, however, involved the fractionation of each crude preparation, and this in many cases revealed the presence of more than one vitamin, with obviously distinct functions, each calling therefore for separate endeavours towards its isolation and purification. It may be mentioned in illustration that yeast, which because it represents a concentrated mass of living cells capable of active growth, and at the same time is available in large amounts, was early and justifiably looked to as a probable source of vitamins, has yielded some of them in a complex which even to-day has perhaps not been fully analysed.

The position of knowledge at the present moment will be made sufficiently clear if the most salient characteristics of each recognised vitamin are very briefly reviewed. Unfortunately, it is impossible at the same time to give credit to the many who have shared in the heavy labours involved in the remarkable recent advances in the subject.

Vitamin $A$. This vitamin is found in association with animal fats and exists in specially high concentration in the livers of fishes. It was discovered and studied in cod liver oil, and at first was not distinguished from vitamin $D$, but by 1922 it had become clear that there were two 'fat soluble' vitamins with functions entirely distinct.

Vitamin A exerts an important influence in the body. In its absence young animals fail to grow. Lack of a proper supply leads to degenerative changes in the epithelial cells which line the outer surfaces of the body, and among the characteristic symptoms which follow upon such a lack is a pathological condition of the eyes known as xerophthalmia. As an independent phenomenon night blindness may occur. Very noteworthy is the evidence which shows that an adequate supply of this vitamin protects against certain types of infection. One of the most interesting advances 
in our knowledge of vitamins is the recent proof that vitamin $\mathrm{A}$ is closely related chemically to the carotenes, a group of yellow pigments widely distributed in plant tissues, and the further proof that carotenes, when they are consumed in green vegetables, are converted in the liver into the vitamin itself. These discoveries have thus shown that vegetable foods are an effective source of the vitamin, and they have also greatly helped in leading to our present knowledge of its actual chemical nature. It has been obtained pure in the form of an oil, and chemical studies have revealed its essential molecular structure.

Vitamin $B_{1}$. This is the vitamin of which a deficiency in the food supply leads as a final issue to the disease beriberi. It exerts a general influence in the body, and would seem to be essential to the normal progress of carbohydrate metabolism, but a specialised aspect of its functions is the maintenance of a normal equilibrium in the nervous tissues. It is widely distributed in natural foods, but in concentrations which vary greatly. We have seen that the circumstance of its presence in the cortical parts of grains and absence from the endosperm led, through the work of Eijkman and his followers, to one of the earliest suggestions for the existence of vitamins. It is relatively abundant in yeast, and this has been the material chiefly used as a source of it for experimental work. Much effort in Great Britain, in particular by $\mathbf{R}$. Peters, has been spent in the effort to obtain it in a pure state, an end which seems now to have been reached. Its actual mole cular structure is not yet known, but its empirical formula is probably $\mathrm{C}_{12} \mathrm{H}_{16} \mathrm{~N}_{4} \mathrm{OS}$. Alone among the known vitamins it contains sulphur in its molecule.

Vitamin $B_{2}$. When yeast extracts were first employed as addenda to deficient diets, their most notable effect, apart from the promotion of growth, seemed to be the prevention of nervous lesions. They were supposed to supply an 'antineuritic' vitamin alone. This is now $B_{1}$. Further studies of such extracts showed, however, that they certainly contain at least one other vitamin more stable towards heat than $B_{1}$, and clearly showing quite different properties. In its absence serious skin lesions develop resembling in animals those seen in the human disease pellagra. There is now indeed little doubt that a prominent factor in the causation of this disease is a lack of this vitamin in the food. It has been labelled $B_{2}$. Quite recently, however, a further complication has come to light in this connexion. Preparations of ' $\mathrm{B}_{2}$ ' as hitherto employed would seem to contain two active factors, one promoting growth without being concerned with skin conditions, and a second to which the 'anti-pellagra' influence is due. The latter is now under intensive study, but its chemical nature is yet unknown. The former, like vitamin $\mathrm{A}$, is related, as shown by the researches of R. Kuhn and P. Karrer, to a group of naturally occurring pigments, but in this case to the flavines. The vitamin is in fact identical with a flavine which is present in milk.

Vitamin $C$. While the prevention and curative influence of foods containing this, the anti scorbutic vitamin, has been so long known, it remained for quite recent research to establish its existence as a definite chemical substance, to produce it pure and to determine its exact chemical nature. It is present in most fresh foods but often only in very small amounts. It is present in greatest concentration in fruits and green vege. tables, but in amounts varying greatly from species to species. Cereal foods contain none. It is characteristically less stable than the other known vitamins, being destroyed when foods are long kept, dried or heated; the influence of oxygen being a potent factor in its destruction. This instability accounts for many chapters in the long history of scurvy and its incidence. Much labour has been spent during recent years in determining quantitatively its distribution in foods and in endeavour to isolate it. Success in the latter aim was reached by A Szent-Gyorgyi three years ago. Its constitution has been fully worked out by W. N. Haworth and his colleagues, revealing the interesting fact that the physiologically potent substance is related to the simple carbohydrates, being a derivative of the hexose sugar gulose. The vitamin is now to be known as ascorbic acid.

Vitamin $D$. This, the anti-rachitic vitamin, is generally associated with vitamin $\mathrm{A}$ in animal fats, and with the latter, is present in exceptionally large amount in fish liver oils. Studies in the etiology of rickets have proved that this disease can be prevented or cured, on one hand by an adequate supply of this vitamin in the food, and, on the other, by adequate exposure of the body to sunlight. A satisfactory explanation of this remarkable relation arrived with the proof that ultra-violet irradiation converts an inactive precursor into the vitamin itself, and that the former is present in the tissues. During the year 1929, owing in particular to the work of Rosenheim and Webster, and that of Hess and Windaus, it was made clear that the substance which on irradiation is activated is ergosterol, which in small amounts is present in most living tissues. As it is therefore present in many natural foods, the anti-rachitic value of these is increased by exposure to rays of suitable wave-length. A preparation of the vitamin made by the irradiation of ergosterol in vitro is known as 'calciferol'. Its potency is remarkable; one ten thousandth of a milligram a day added to 
a rickets-producing diet will in a rat entirely prevent the appearance of the disorder. In the case of a child the effective daily dose is a very small fraction of a grain. The rigorous proof that lack of a fat-soluble vitamin is responsible for the induction of rickets was furnished by the classical experiments of $\mathrm{E}$. Mellanby begun twenty years ago. More recently, the importance of vitamin $\mathrm{D}$ in the processes of normal dentition has been shown by May Mellanby.

Vitamin $E$. In 1922 it was first shown by H. M. Evans and K. S. Bishop that a vitamin, distinct from others then known, is essential for successful reproduction. It has been termed the antisterility vitamin, but this term implies functions more specific than those which are actual. Deprivation of vitamin $\mathrm{A}$, for example, will ultimately lead to failure in reproduction. Nevertheless, the influence of vitamin $\mathrm{E}$ (now so-called) is exerted on specific lines. In its absence, there is degeneration of the testes in the male and a failure of the placental functions in the female. The richest sources of this vitamin so far discovered are certain green vegetables and wheat embryos. It is, however, widely distributed in food-stuffs, and as it is active in very small amounts, the possibility of any lack of it can seldom arise. Its constitution is unknown.

These very brief descriptions of the known vitamins leave out, of course, a multiplicity of facts which have been discovered concerning each of them, and omit reference to the work of very many investigators. They may serve, however, to indicate the lines on which vitamin research has hitherto progressed.

Characteristic of each vitamin is the very small amount in which it exercises its physiological functions, and the circumstance that all are present in very low concentrations in the materials from which they have to be separated has greatly added to the difficulties of their study. It will be admitted, however, that we have now a sound body of knowledge concerning them, establishing their nutritional importance and throwing no little light on their nature. Research in the field is now receiving much help on its constitutional side from modern physical methods, and on its biological side from increasing interest on the part of a large number of clinicians. Vitamin therapy is now joining hands with endocrine therapy, and the League of Nations Permanent Commission on Biological Standards has recognised its growing importance by accepting standards for measures of vitamin activity and defining units in terms of such standards.

Some at least of the conditions which are now grouped as deficiency diseases are of world-wide importance, and though the clear-cut symptoms which the experimentalist can observe in animals under strictly controlled conditions are often obscured by intercurrent infections or other complications in clinical cases, yet, once a food deficiency has been recognised as an essential link in the chain of causation, the method of cure becomes in every case as certain as it is logical. On the other hand, once the hygienist has become convinced that this or that disease is really due to faults in the diet of communities, its prevention, with or without administrative action, should be easy to secure. Although a defect in the supply of a vitamin, if serious and continued, may result in actual disease, it is, in Great Britain, more important to realise that a sub-optional supply of any essential food constituent cannot fail to induce sub-normal health which, especially when induced in childhood, may leave permanent disability.

Apart from its own inherent importance, the revelation of the significance of vitamins can fairly be said to have directed closer attention to the nutritional importance of other minor constituents of natural foods. The specific needs of the body are proving to be numerous, and lack of materials called for in very small amounts are proving to be just as important to final issues in nutrition as are those required in much larger amounts. This applies to the mineral as well as to the organic constituents of food, and ill-assorted diets may be deficient in the former no less than in the latter.

For the progress of scientific knowledge concerning these needs, each separate factor has called, and continues to call, for separate and intensive study ; but the demands of right nutrition need to be viewed as a whole. We need to know what should be the ideal balance among the many essentials, and how best to secure that it shall be approached in the food supply of all classes of the community. Short of this, we have to-day sufficient knowledge to be sure that malnutrition in its subtler aspects often accounts for disabilities which have hitherto been ascribed to constitutional defects or to other circumstances. With present knowledge, moreover, it should be easy, economic questions apart, to prevent such malnutrition everywhere. There is almost sufficiency in the statement that certain foods often held to be luxuries have to be recognised as necessities for all. Recognition of this bears upon all the problems of a national food supply; upon production, preservation, transport and distribution.

It is interesting to remember that the effective development of the recent knowledge concerning the more subtle aspects of nutrition has been almost co-terminous with the reign of King George. 